\title{
Facile Biosynthesis and Antioxidant Property of Nanogold-Cellulose Fiber Composite
}

\author{
Nattinee Bumbudsanpharoke, Jungwook Choi, Insik Park, and Seonghyuk Ko \\ Department of Packaging, Yonsei University, Wonju 220-710, Republic of Korea \\ Correspondence should be addressed to Seonghyuk Ko; s.ko@yonsei.ac.kr
}

Received 30 March 2015; Revised 19 May 2015; Accepted 31 May 2015

Academic Editor: Renyun Zhang

Copyright (C) 2015 Nattinee Bumbudsanpharoke et al. This is an open access article distributed under the Creative Commons Attribution License, which permits unrestricted use, distribution, and reproduction in any medium, provided the original work is properly cited.

\begin{abstract}
Direct synthesis of gold nanoparticles (AuNPs) on cellulose fiber has been successfully performed via facile green approach using lignin-containing unbleached kraft softwood pulp. The resulting AuNPs composited fibers showed apparent color change from pale yellow to purplish-dark brown by varying the amount of gold ions $\left(\mathrm{Au}^{3+}\right)$ due to the surface plasmon resonance of nanogold. Further confirmation of AuNP formation on the fiber surface was conducted by UV-Vis diffuse reflectance spectroscopy (DRS), $\mathrm{X}$-ray diffraction (XRD), and field emission scanning electron microscopy (FE-SEM). X-ray photoelectron spectroscopy (XPS) analysis revealed that gold nanoparticles formed on the fiber are well-defined pure metallic gold, indicating that $\mathrm{Au}^{3+}$ ions are efficiently bioreduced into $\mathrm{Au}^{0}$ and bind to the fiber surface. Antioxidant activity was evaluated by decomposition of 2,2-diphenyl1-picryl-hydrazyl (DPPH) in dark and light condition. As-prepared unbleached kraft fiber-AuNP composite showed significantly enhanced antioxidant activity and its DPPH scavenging rate reached about $86.05 \%$.
\end{abstract}

\section{Introduction}

Nowadays the contemporary consumers are not purchasing only a product, but a solution for healthier and safer food [1]. The shelf life of fatty foods and dry grains is profoundly affected by the formation of active free radicals (e.g., reactive oxygen species). Reduction or termination of the lipid oxidation provides longer shelf life product and traditionally, antioxidants are coated on food surface or mixed into initial food formulations. However, lack of selectivity to the food surface and the side reactions between food system and antioxidant active compounds limit the use of these approaches [2]. Antioxidant active packaging alternatively plays a crucial role in inhibiting the oxidative rancidity in food by scavenging the free radicals generated by the oxidative processes.

Gold nanoparticle (AuNP), a human trace element, has gained much more attention and tremendous works in pharmacological sectors and biomedical applications due to its unique therapeutic activity, being inert, and nontoxic nature [3]. AuNP has drawn enormous attention as one of the most effective heterogeneous catalysts, particularly, for selective oxidation $[4,5]$. There is also considerably interest in using AuNP for both in vitro and in vivo antioxidant application [6-8]. BarathManiKanth et al. [9] disclosed the effectual role of AuNP as an antioxidative agent for lipid peroxidation and reactive oxygen species in diabetic mice. Balasubramani et al. [10] showed that AuNP is highly capable of scavenging free radicals which lead to anticancer property. This study proposed an alternative application of AuNP as a drug in nanomedicine to treat breast cancer in the future. Moreover, Leu et al. [11] investigated the effects of AuNP combined with epigallocatechin gallate and $\alpha$-lipoic acid in wound healing. They found that the treatment of cutaneous wound in mice can be accelerated by antioxidation effect of AuNP compound. Therefore, regarding unique antioxidation characteristic of nanogold, the advancements in use of AuNP for potential antioxidant food packaging may bring new innovative to active packaging.

A wide range of methods have been developed to produce AuNP in a multitude of chemical and physical processes $[12,13]$. The traditional and most widely used method for the synthesis of AuNP commonly involves the reduction of a soluble metal salt by a reducing agent or 
high-temperature gaseous conditions. Hydrazine, tri-sodium citrate, sodium borohydride, and dimethylformamide are examples of reported reducing and stabilizing agents [14, 15]. However, exposure to these highly reactive chemicals poses potential risks to human health and the environment [16].

Enormous efforts have been made to integrate green chemistry concepts to metal nanoparticle syntheses, without accumulating an enormous quantity of toxic chemicals in the environment $[17,18]$. Natural resources such as plant extracts, microorganisms, biodegradable polymers, and polysaccharides are known to be effective biological materials for alternative green syntheses of metallic nanoparticles [19, 20]. Among the various greener reagents, polysaccharides are quite advantageous when utilized in green synthesis due to the fact that they have an abundance of hydroxyl groups and hemiacetal reducing ends; thus, they can be used as both reducing and stabilizing agents for metal nanoparticles [21]. Various polysaccharides such as buffered glucose incorporated with starch [22], chitosan associated with organic acid [23], cellulose in ionic liquid [24], guar gum [25], dextran [26], pullulan [27], and arabinoxylan [28] have been employed for AuNP synthesis.

More recently, there has been considerable interest in the direct synthesis of metal nanoparticles on fibers, since gold colloids easily aggregate to yield bulkier materials, which in turn lowers the optical and catalytic activities [29]. Zhang et al. [30] developed AuNPs embedded in a biotemplate using a single-step method in which AuNPs were synthesized by polyethyleneimine and simultaneously coated on bacteria cellulose. Johnston and Nilsson [31] have proposed a green reduction mechanism and immobilization of nanosilver and nanogold using unbleached lignin-containing paper fibers. Aromatic methoxy and phenol groups of lignin were found to reduce $\mathrm{Au}^{3+}$ to $\mathrm{Au}^{0}$ and to firmly bind AuNPs to the fiber surface. In addition, Azetsu et al. [32] demonstrated the direct synthesis of AuNPs by TEMPO-oxidized softwood kraft paper. The aldehydic moieties in carbohydrates were reported as a reducing agent, while obtained AuNPs were deposited on cellulose fibers.

Despite several pathways of AuNP green synthesis, there is still great interest in developing a simple and sustainable method to prepare well-defined gold nanoparticles on renewable cellulose-based fibers without the use of additional reducing chemicals and fiber modification. To date, few studies have focused on such techniques. Here we report a facile biosynthesis of AuNP using unbleached kraft softwood fiber which acts as both reducing-stabilizing agent and substrate for the AuNP immobilization. UV-Vis diffuse reflectance spectroscopy (DRS), X-ray diffraction (XRD), field emission scanning electron microscopy (FE-SEM), energy dispersive spectroscopy (EDS), and X-ray photoelectron spectroscopy (XPS) were used to characterize the resulting fiber-AuNPs composite. The antioxidant activity of the AuNPs composite sheets was investigated by measuring the scavenging rate of 2,2-diphenyl-1-picryl-hydrazyl (DPPH) as an indicator.

\section{Experimental}

2.1. Materials. ACS reagent grade hydrogen tetrachloroaurate(III) trihydrate $\left(\mathrm{HAuCl}_{4} \cdot 3 \mathrm{H}_{2} \mathrm{O}\right.$, chloroauric acid) was used as received from Alfa Aesar (Ward Hill, IL, USA) in the preparation of AuNPs on the fiber. Unbleached softwood kraft pulp was obtained from Dongli Paper (Ansan, Korea). 2,2-Diphenyl-1-picryl-hydrazyl (DPPH) was purchased from Sigma Chemical Co. (St. Louis, MO, USA). Ultra-pure water with a specific resistivity of $18 \mathrm{M} \Omega \cdot \mathrm{cm}$ was used in this study.

2.2. Biosynthesis of AuNP on Unbleached Kraft Pulp. AuNPs composite fibers were simply prepared by a greener route using unbleached kraft pulp. An aqueous solution of chloroauric acid as the AuNP precursor was prepared at various concentrations ranging up to $40 \mathrm{mM}$ by dissolving $\mathrm{HAuCl}_{4} \cdot 3 \mathrm{H}_{2} \mathrm{O}$ in ultra-pure water. The unbleached softwood kraft pulp was first disintegrated with a valley beater according to the Technical Association of the Pulp and Paper Industry (TAPPI) test method T 200 sp-01 [33]. The pulp was then mechanically refined to approximately $430 \mathrm{~mL}$ of Canadian Standard Freeness (CSF) according to TAPPI test methods T 227 om-04 [34] and T $200 \mathrm{sp}-01$ prior to biosynthesis of nanogold. In a typical synthesis of AuNPs on the fibers, $2 \mathrm{~g}$ of refined wet pulp with approximately $90 \%$ moisture content was suspended in $40 \mathrm{~mL}$ of chloroauric acid aqueous solution with constant stirring until it was completely dispersed ( $0.5 \%$ consistency, $0.5 \mathrm{~g}$ dried pulp/L). Following preparation of the pulp suspension, in order to facilitate AuNP synthesis via reduction of $\mathrm{Au}^{3+}$ to $\mathrm{Au}^{0}$, the mixture of pulp fibers and chloroauric acid was subsequently placed in an autoclave at $15 \mathrm{psi}$ and $121^{\circ} \mathrm{C}$ for $30 \mathrm{~min}$. After the reaction was complete, AuNPs composite cellulose fibers were centrifuged and rinsed repeatedly with DI water followed by suctioning on a 200-mesh wire to form a wet sheet. The obtained fiber-AuNPs composite sheet was dried at $60^{\circ} \mathrm{C}$ under vacuum overnight. Finally a purplish to red-brownish colored AuNPs conjugated papers were obtained. Paper made of only unbleached kraft pulp, denoted by unbleached kraft paper (UBK), was also prepared for use as a reference sample. In order to quantify the amount of $\mathrm{Au}$ synthesized on cellulose fiber, the EDS analysis on the surface of as-prepared UBK-AuNPs composites has been performed. As a result, amount of Au was confirmed as $1.9 \mathrm{wt} \%$ and $5.3 \mathrm{wt} \%$.

\subsection{Characterization of Cellulose-AuNP Composite. UV-Vis} diffuse reflectance spectra were recorded on a UV-Vis spectrophotometer (V-600, Jasco, Japan) with an attached integrating sphere. A barium sulfate $\left(\mathrm{BaSO}_{4}\right)$ pellet was used as a reference to measure absorption of as-prepared cellulose fiber-AuNP composite sheets. X-ray diffraction patterns were obtained with a Bruker D2 Phaser with a Ni-filtered $\mathrm{Cu} \mathrm{K} \alpha$ radiation source $(\lambda=0.15418 \mathrm{~nm})$ using a $1 \mathrm{~mm}$ equatorial slit and $3 \mathrm{~mm}$ air scattering slit. The surface morphology of the composite sheets was observed by field emission scanning electron microscopy (FESEM Quanta FEG 250, FEI Co., Ltd., USA) in backscattered electron (BE) mode after sputtering $\mathrm{Pt} / \mathrm{Pd}$ onto the specimen. FTIR analyses were performed using a Spectrum 65 FTIR (Perkin Elmer, USA). Each spectrum was collected in attenuated total reflectance (ATR) mode with $2 \mathrm{~cm}^{-1}$ resolution for 16 scans in the wavenumber range of $4000-400 \mathrm{~cm}^{-1}$. XPS data were obtained using a Kratos AXIS ULTRA XPS system with a monochromated 


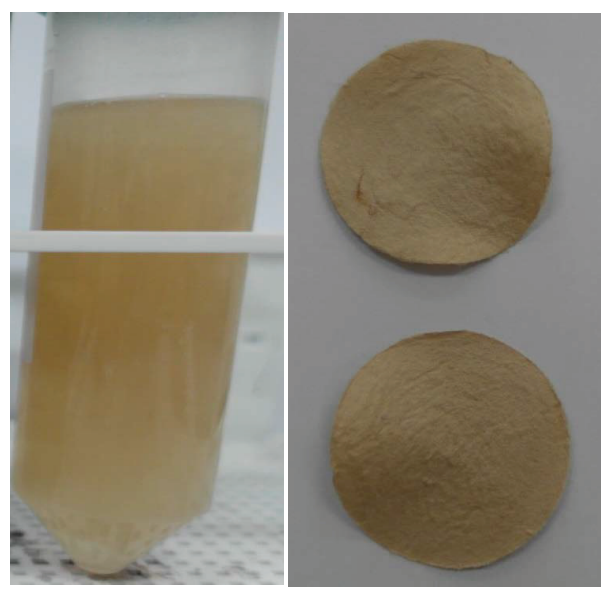

(a)
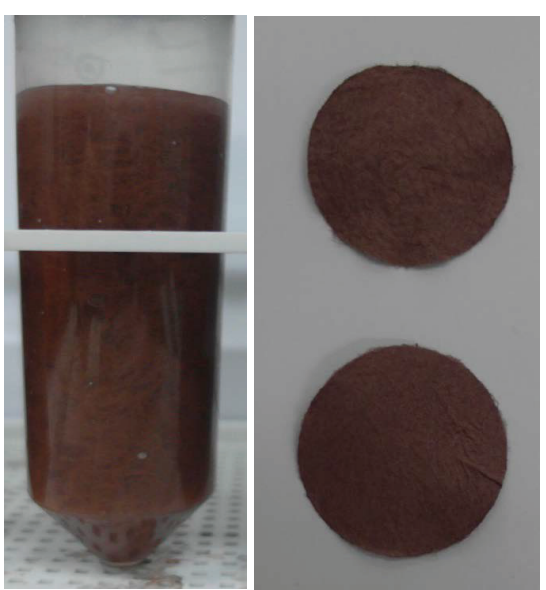

(b)
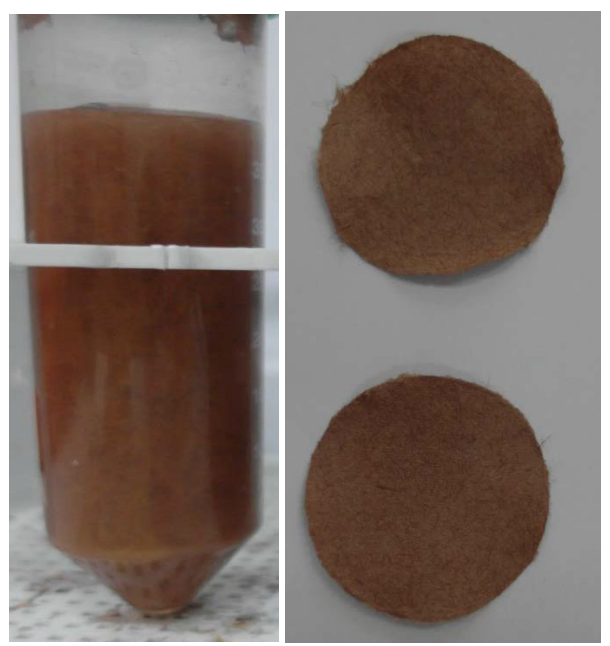

(c)

Figure 1: Photographs of unbleached kraft fibers and composite sheets after formation of gold nanoparticles with different concentrations of $\mathrm{HAuCl}_{4} \cdot 3 \mathrm{H}_{2} \mathrm{O}$; (a) UBK only; (b) UBK-Au (1.9 wt\%); (c) UBK-Au (5.3 wt\%).

$\mathrm{Al} \mathrm{K} \alpha \mathrm{X}$-ray source. Samples were mounted in the sample chamber on double-sided copper tape $(3 \mathrm{M})$ at an ultra-high vacuum (UHV) chamber operating pressure of $10^{-8}$ Torr. To investigate the surface chemistry of both the reference and UBK-AuNPs composite, survey and high-resolution spectra were processed using CasaXPS software from which a Shirley background was subtracted before fitting the spectra peaks to a symmetric Gauss-Lorentz sum function. All spectra were calibrated to the binding energy of $\mathrm{C}$ 1s photoelectrons at $284.6 \mathrm{eV}$.

2.4. Evaluation of Antioxidant Property. Cellulose fiberAuNP composite sheets were subjected to the determinations of the DPPH radical scavenging ability in both light and dark condition. Samples were cut into small pieces (approximately $3.0 \times 3.0 \mathrm{~mm}$ ) followed by immersing in $5.0 \mathrm{~mL}$ of dark purple-colored DPPH solution $(100 \mu \mathrm{mol} / \mathrm{L}$ in absolute ethanol). It was then placed and constantly shaken on a twist shaker (TW3, FINEPCR, Seoul, Korea) at ambient temperature for $180 \mathrm{~min}$. Three replicates and UBK as control sample were prepared for each condition. The radical scavenging rate was measured with the absorbance of DPPH at $516 \mathrm{~nm}$ wavelength on UV-Vis spectrum. The antioxidant activity was calculated with the following equation:

$$
\text { Scavenging }(\%)=\left[\frac{A_{\text {initial }}-A_{\text {sample }}}{A_{\text {initial }}}\right] \times 100,
$$

where $A_{\text {initial }}$ was the absorbance of the fresh DPPH solution and $A_{\text {sample }}$ was the absorbance of the test sample at reaction time, $180 \mathrm{~min}$.

\section{Results and Discussion}

This study demonstrated a simple green reduction method to prepare cellulose fiber-AuNP nanocomposites using unbleached kraft pulp. During the reduction of $\mathrm{Au}^{3+}$ by lignin-containing unbleached cellulose fibers, the color of the pulp slurry obviously changed and progressively deepened with increasing chloroauric acid concentration. Figure 1 shows digital images of composite sheets along with the pulp slurry, which presents the apparent change in color of fibers from pale brown to purple and wine-red brown as the 
concentration of chloroauric acid was increased. This diverse color change is attributed to the surface plasmon resonance (SPR) of AuNPs [35-37], implying the direct formation of nanogold on the fiber surface.

Figure 2 presents the UV-Vis diffuse reflectance spectra of as-prepared UBK-AuNPs composite sheets. Unbleached kraft paper was used as a comparative reference, which showed broad absorption in the UV region below $400 \mathrm{~nm}$. All of the cellulose fiber-AuNPs composite spectra exhibited a strong absorption peak at $539 \mathrm{~nm}$ corresponding to a characteristic SPR absorption band of gold nanoparticles. The peak was more symmetric and narrower with increasing concentrations of gold precursor, which indicates an increase in particle size and growth of AuNPs. It can also be inferred that the Au nanoparticles formed on cellulose fibers by the in situ biosynthesis were dispersed on the surface of the fibers, since with a higher dispersion of metal nanoparticles more interactions occur between particles, which contribute to the enhancement of surface plasmon absorption. Previous studies have reported similar effects on particle size and polymodal size distribution depending on the amount of chloroaurate ions involved in nanogold synthesis using plant extracts $[38,39]$.

The microstructure of UBK-AuNPs composites was characterized by FESEM in BE mode, and images are shown in Figure 3. Due to the distinctive difference in contrast, gold nanoparticles can be readily identified as white dots dispersed over the fibers. The images confirm that gold nanoparticles were successfully formed and decorated the surface of the fibers. As clearly observed in Figure 3(c), gold nanoparticles had a predominately spherical shape with sizes of less than $100 \mathrm{~nm}$ in diameter, which was found to be the most common morphology along with sparse rod-shaped and triangular plates. The images distinctly indicate that incremental number and surface coverage of AuNPs on fiber can be achieved simply by varying the concentration of the chloroauric acid solution.

Figure 4 shows XRD patterns of various AuNPs composited fiber sheets and unbleached kraft paper. Unbleached kraft paper exhibited diffraction peaks at $2 \theta=17.3^{\circ}, 23.1^{\circ}$, and $34.9^{\circ}$, which are typical for crystalline cellulose I [40]. By contrast, fiber-AuNPs composite sheets had not only characteristic peaks corresponding to the crystalline cellulose I, but also four sharp peaks at $2 \theta=38.6^{\circ}, 44.9^{\circ}, 65.0^{\circ}$, and $78.0^{\circ}$, which are indexed to the (111), (200), (220), and (311) planes of the face-centered cubic (fcc) lattice of crystalline $\mathrm{Au}$ (JCPDS number 04-0784) [41]. This result suggests that Au nanocrystals were effectively produced while the intrinsic crystalline structure of the cellulose matrix remained intact. It is also evident that as more chloroaurate ions were involved in the preparation, the Au diffraction peaks gradually increased and became sharper, indicating particle growth and an increase in particle size in accordance with gradual sharpness and blue-shift of the surface plasmon absorption as discussed in Figure 2. The average particle size of each fiber-nanogold composite was calculated from the X-ray line broadening, using the Scherrer equation [42] as follows:

$$
\tau=0.9 \frac{\lambda}{\beta \cos \theta_{B}},
$$

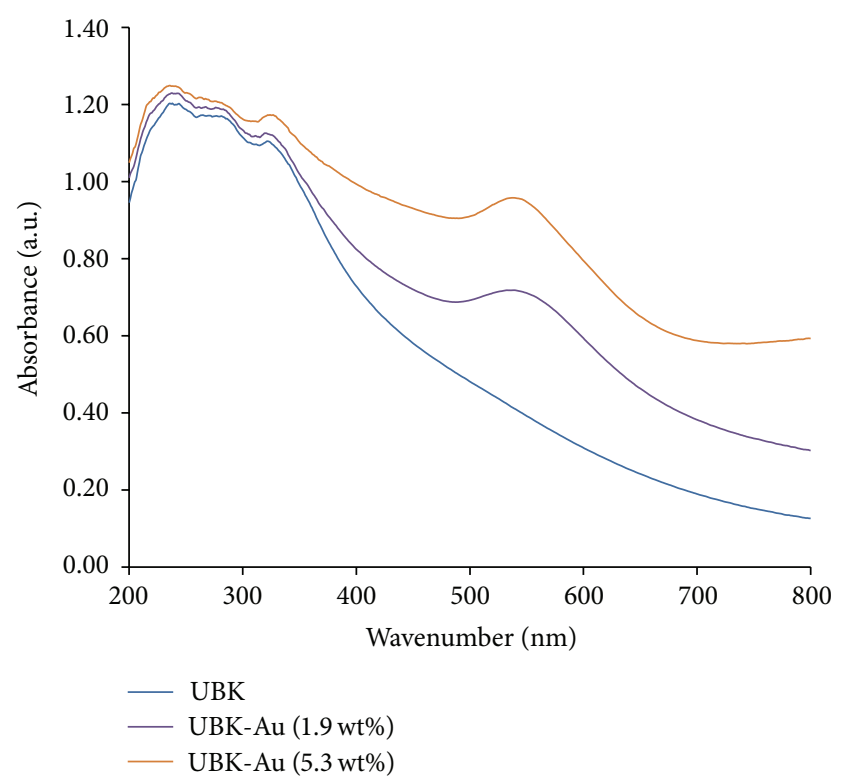

FIGURE 2: UV-Vis diffuse reflectance spectra of UBK-AuNPs composite sheets.

where $\tau$ is the mean diameter of the particle (nm), $\lambda$ is the wavelength of the $\mathrm{X}$-ray used, $\beta$ is the full width of diffraction lines at half of maximum intensity (radians), and $\theta_{B}$ is the diffraction angle corresponding to the maximum peak. Based on this formula, average size of AuNP was estimated to be $12.4,16.4$, and $21.0 \mathrm{~nm}$ in order of increasing chloroauric acid concentration.

The presence of $\mathrm{Au}$ and Au-related compounds on the fibers was also studied by XPS. Survey scan analyses of UBK paper and the UBK-AuNPs composite are presented in Figure 5. As seen in Figure 5, an additional peak corresponding to Au appeared with the as-prepared nanogold composite sheet, which was absent in the unbleached kraft paper spectrum. In addition, it was observed that the ratio of $\mathrm{C}$ and $\mathrm{O}$ in the final composite slightly increased from 0.48 to 0.5 , which may be attributed to the participation of cellulose in gold synthesis and, in part, thermal degradation. Inset in Figure 5 shows the XPS spectrum of the $\mathrm{Au} 4 \mathrm{f}$ region, which can be well fit by two peaks centered at $\mathrm{BE}$ positions of $84.1 \mathrm{eV}$ and $87.8 \mathrm{eV}$ assigned to the $\mathrm{Au} 4 \mathrm{f}_{7 / 2}$ and $\mathrm{Au} 4 \mathrm{f}_{5 / 2}$ core levels of metallic gold $\left(\mathrm{Au}^{0}\right)$, respectively [43]. No peaks corresponding to $\mathrm{Au}^{3+}$ (85.8 and $89.5 \mathrm{eV}$ ) [44] or Au-O species $(85.5 \mathrm{eV})$ [45] were observed. This result is in good agreement with the XRD characterization and provides further evidence that gold ions $\left(\mathrm{Au}^{3+}\right)$ were successfully reduced to metallic pure $\mathrm{Au}$ that bound to the cellulose fibers. The XPS peak for C 1 s at $\mathrm{BE}=284.6 \mathrm{eV}$ ascribed to adventitious carbon was used to correct the binding energy shift produced by the sample.

Figure 6 shows FTIR spectra of unbleached kraft paper and cellulose fiber-AuNP composite sheets. No distinguishing peaks were observed between samples except those in the region between 1550 and $1650 \mathrm{~cm}^{-1}$, which were assigned to the aromatic skeleton vibration of lignin, conjugated $\mathrm{C}=\mathrm{O}$ stretching of the phenolic group, and $\mathrm{C}-\mathrm{O}$ stretching from 


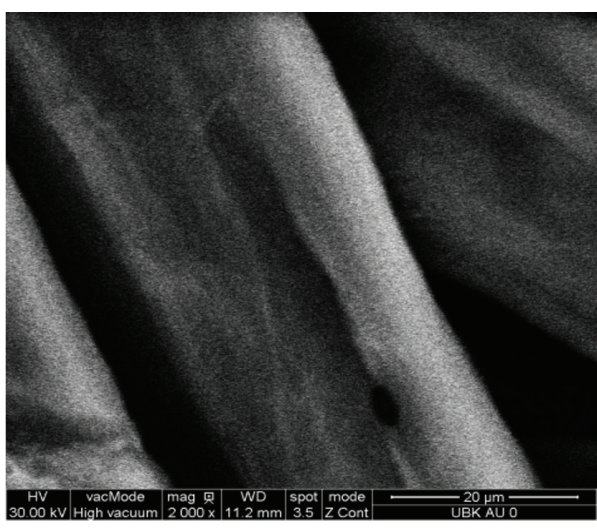

(a)

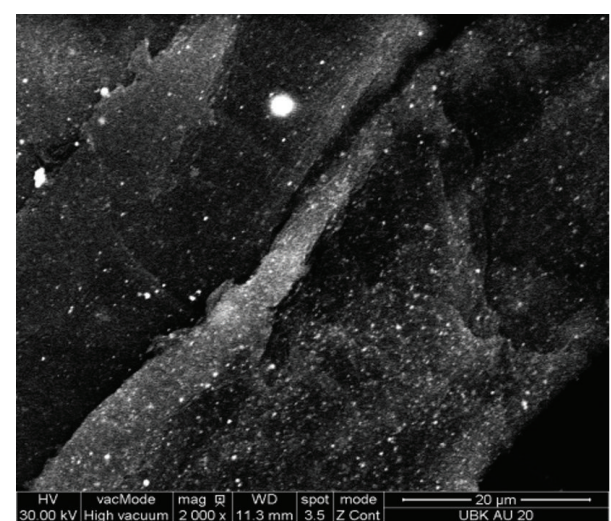

(b)

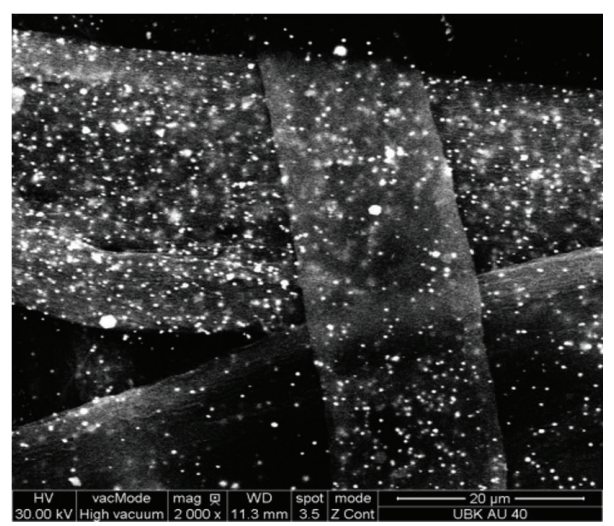

(c)

FIGURE 3: FESEM images in backscattered electron mode of (a) UBK, (b) UBK-Au (1.9 wt\%), and (c) UBK-Au (5.3 wt\%) nanocomposites.

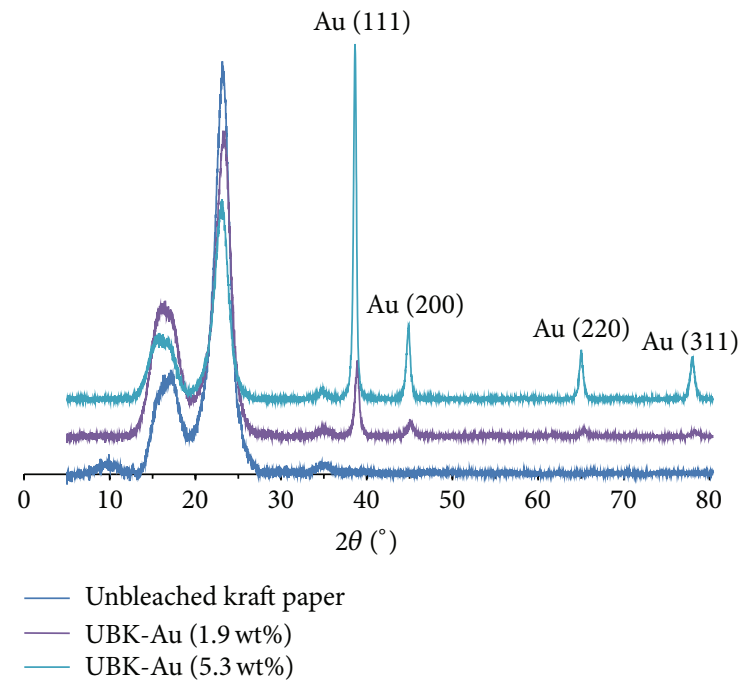

FIGURE 4: XRD patterns of (a) UBK, (b) UBK-Au (1.9 wt\%), and (c) UBK-Au (5.3 wt\%) nanocomposites.

lignin $[46,47]$. In particular, a reduction in the intensity of the peak at 1580 to $1585 \mathrm{~cm}^{-1}$, which is associated with the $\alpha$-carbonyl group on lignin, is compatible with oxidation of the clustering and movement of $\mathrm{Au}^{0}$ species resulting from $\mathrm{Au}^{3+}$ bioreduction occurring in the electron rich lignin component. This result is indicative of participation of the lignin component in AuNP synthesis. A similar result was reported by $\mathrm{Li}$ et al., indicating that the $\mathrm{C}=\mathrm{O}$ vibration peak changed slightly with a higher concentration of silver ion [48]. Johnston and Nilsson recently reported that nanogold and nanosilver can be formed and bind directly to the surface of paper fibers, and for this synthesis, phenol and aromatic methoxy groups of lignin are essential to reduce $\mathrm{Au}^{3+}$ to $\mathrm{Au}^{0}$ and $\mathrm{Ag}^{+}$to $\mathrm{Ag}^{0}$, respectively [31].

Figure 7 illustrates the DPPH radical scavenging rate of cellulose fiber-Au nanocomposites under light and dark condition and surface morphology of composite sheets used in this study. UBK was also tested to examine DPPH adsorption and to obtain pure AuNP effect on radical scavenging in UBK-AuNP samples. As seen in Figure 7(a), DPPH adsorbed by UBK and its efficiency was about $47.7 \%$ of initial DPPH concentration. In contrast, UBK-Au nanocomposites showed significantly improved DPPH radical scavenging capacity in both the presence and absence of light. Interestingly, when cellulose-AuNP nanocomposite was exposed to light, the scavenging rate $(86.05 \% \pm 0.009)$ was slightly higher than that from the dark condition $(77.86 \% \pm 0.006)$, indicating AuNP becomes more sensitive to quench reactive radicals by light 


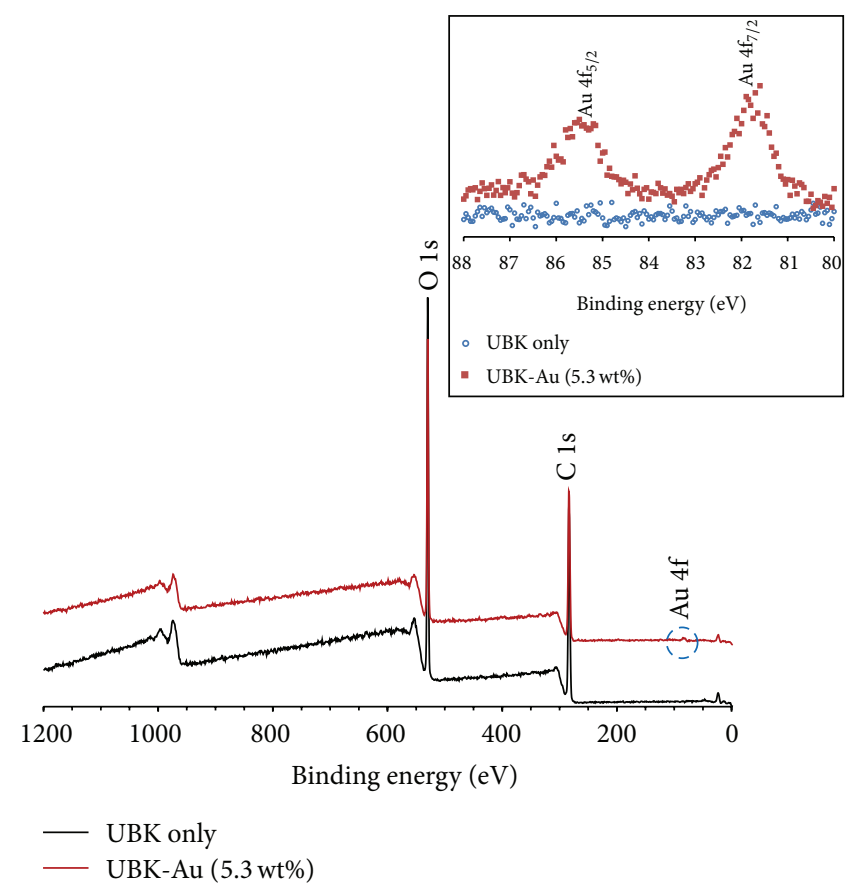

FIGURE 5: Representative XPS survey scan spectra of UBK only and UBK-Au (5.3 wt\%) nanocomposite. Inset shows Au $4 \mathrm{f}$ region spectrum consisting of $\mathrm{Au} 4 \mathrm{f}_{5 / 2}$ and $\mathrm{Au} 4 \mathrm{f}_{7 / 2}$.

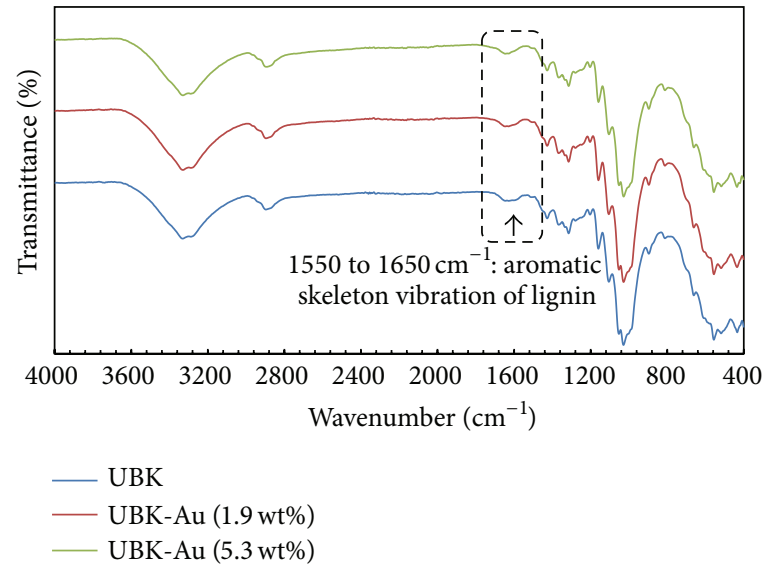

FIGURE 6: FTIR spectra of UBK and UBK-AuNPs composite sheets.

assisted activation $[49,50]$. It seems reasonable to assume that adsorption occurred first mostly by fibers and voids in the layered fiber matrix and subsequently contributes to enhancing the AuNP radical scavenging. As seen in the micrographs the fibers formed dense fiber network structures resulting from internally fibrillated and flexible fibers. Compared to UBK sheet, UBK-AuNP composite sheet has a somewhat loose configuration and was found to contain many large pores between fibers. As a result, microscale voids formed from layered fiber network improve the adsorption affinity of DPPH molecules of UBK-AuNP composite. Improved adsorption capacity synergistically contributes to increased antioxidant performance with gold nanoparticles.

\section{Conclusions}

Cellulose fiber-AuNPs composites have been prepared by a one-step direct synthesis of gold nanoparticles on unbleached softwood cellulose fibers and were investigated for enhanced antioxidant property under ambient condition. It was found that $\mathrm{Au}$ nanoparticles were successfully bioreduced to $\mathrm{Au}^{0}$ from $\mathrm{Au}^{3+}$ without external reducing reagents and were identified metallic gold according to XPS scan of $\mathrm{Au} 4 \mathrm{f}$ region. It was further observed that, thorough analyses by UVVis DRS, FESEM, and XRD, nanoscaled gold particles were well-conjugated directly onto the fiber surface. Antioxidant activity of AuNP composited fiber in scavenging DPPH 


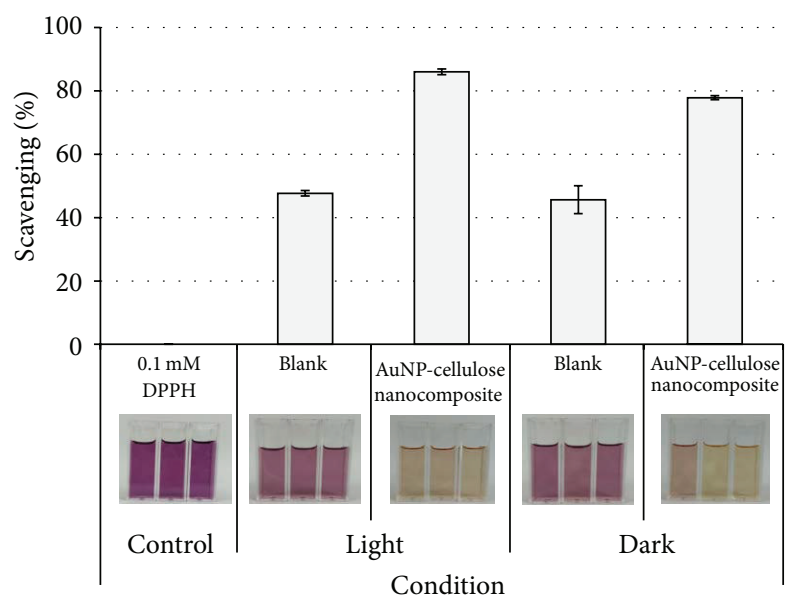

(a)
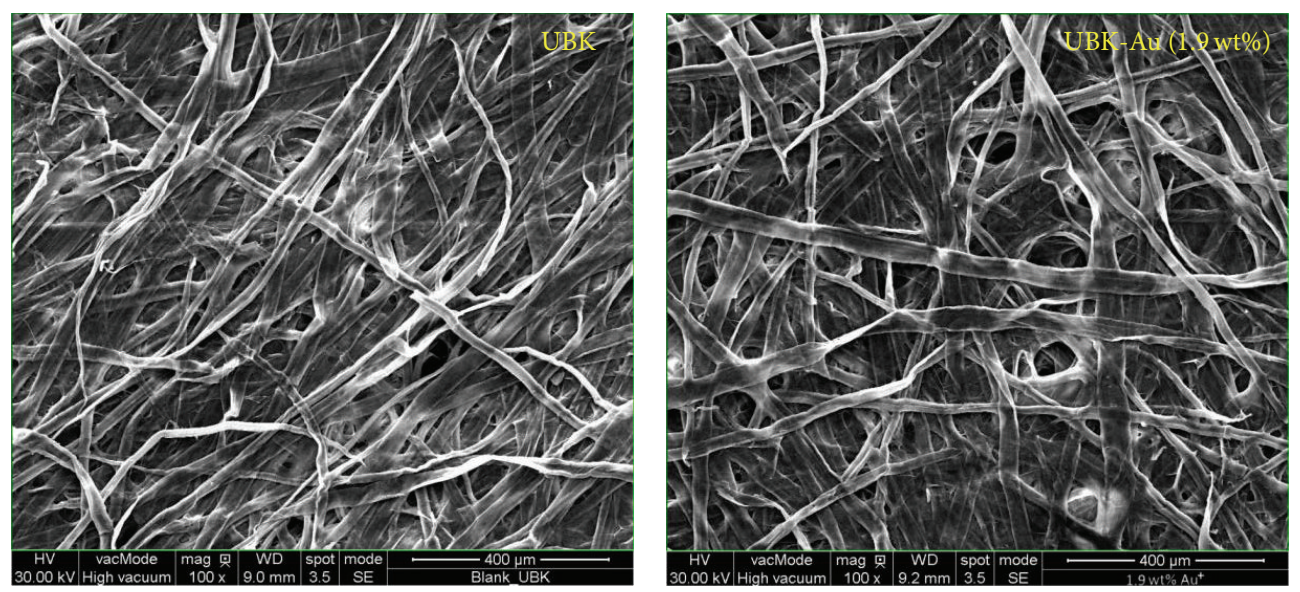

(b)

FIGURE 7: (a) The DPPH scavenging effects and (b) FESEM images of UBK and UBK-Au (1.9 wt\%) composite sheet.

solution was much higher than that of pure fiber composites due to the catalytic function of AuNPs and this result poses possible application for the antioxidant food packaging.

\section{Conflict of Interests}

The authors declare that there is no conflict of interests regarding the publication of this paper.

\section{Acknowledgments}

This study was carried out with the support of "Forest Science and Technology Projects (Project no. S111313L030120)" provided by the Korea Forest Service and the Basic Science Research Program through the National Research Foundation of Korea (NRF) funded by the Ministry of Education (Grant no. 2013R1A1A2060023).

\section{References}

[1] J. Gómez-Estaca, C. López-de-Dicastillo, P. Hernández-Muñoz, R. Catalá, and R. Gavara, "Advances in antioxidant active food packaging," Trends in Food Science \& Technology, vol. 35, no. 1, pp. $42-51,2014$.
[2] S. Gemili, A. Yemenicioğlu, and S. A. Altinkaya, "Development of antioxidant food packaging materials with controlled release properties," Journal of Food Engineering, vol. 96, no. 3, pp. 325$332,2010$.

[3] M. S. Khan, G. D. Vishakante, and H. Siddaramaiah, "Gold nanoparticles: a paradigm shift in biomedical applications," Advances in Colloid and Interface Science, vol. 199-200, pp. 4458, 2013.

[4] M. Stratakis and H. Garcia, "Catalysis by supported gold nanoparticles: beyond aerobic oxidative processes," Chemical Reviews, vol. 112, no. 8, pp. 4469-4506, 2012.

[5] S. Aswathy Aromal and D. Philip, "Facile one-pot synthesis of gold nanoparticles using tannic acid and its application in catalysis," Physica E: Low-Dimensional Systems \& Nanostructures, vol. 44, no. 7-8, pp. 1692-1696, 2012.

[6] B. E. Naveena and S. Prakash, "Biological synthesis of gold nanoparticles using marine algae Gracilaria corticata and its application as a potent antimicrobial and antioxidant agent," Asian Journal of Pharmaceutical and Clinical Research, vol. 6, no. 2, pp. 179-182, 2013.

[7] P. M. Tiwari, K. Vig, V. A. Dennis, and S. R. Singh, "Functionalized gold nanoparticles and their biomedical applications," Nanomaterials, vol. 1, no. 1, pp. 31-63, 2011. 
[8] P. Manivasagan, M. S. Alam, K. H. Kang, M. Kwak, and S. K. Kim, "Extracellular synthesis of gold bionanoparticles by Nocardiopsis sp. and evaluation of its antimicrobial, antioxidant and cytotoxic activities," Bioprocess and Biosystems Engineering, vol. 38, no. 6, pp. 1167-1177, 2015.

[9] S. BarathManiKanth, K. Kalishwaralal, M. Sriram et al., "Antioxidant effect of gold nanoparticles restrains hyperglycemic conditions in diabetic mice," Journal of Nanobiotechnology, vol. 8, article 16, 15 pages, 2010.

[10] G. Balasubramani, R. Ramkumar, N. Krishnaveni et al., "Structural characterization, antioxidant and anticancer properties of gold nanoparticles synthesized from leaf extract (decoction) of Antigonon leptopus Hook. \& Arn," Journal of Trace Elements in Medicine and Biology, vol. 30, pp. 83-89, 2015.

[11] J. G. Leu, S. A. Chen, H. M. Chen et al., "The effects of gold nanoparticles in wound healing with antioxidant epigallocatechin gallate and alpha-lipoic acid," Nanomedicine: Nanotechnology, Biology, and Medicine, vol. 8, no. 5, pp. 767-775, 2012.

[12] P. Alexandridis, "Gold nanoparticle synthesis, morphology control, and stabilization facilitated by functional polymers," Chemical Engineering \& Technology, vol. 34, no. 1, pp. 15-28, 2011.

[13] R. Sardar, A. M. Funston, P. Mulvaney, and R. W. Murray, "Gold nanoparticles: past, present, and future," Langmuir, vol. 25, no. 24, pp. 13840-13851, 2009.

[14] P. Raveendran, J. Fu, and S. L. Wallen, "Completely 'green' synthesis and stabilization of metal nanoparticles," Journal of the American Chemical Society, vol. 125, no. 46, pp.13940-13941, 2003.

[15] R. J. Pinto, M. C. Neves, C. P. Neto, and T. Trindade, "Composites of cellulose and metal nanoparticles," in NanocompositesNew Trends and Developments, F. Ebrahimi, Ed., pp. 73-96, InTech, Rijeka, Croatia, 2012.

[16] F. He, J. Liu, C. B. Roberts, and D. Zhao, “One-step 'green' synthesis of Pd nanoparticles of controlled size and their catalytic activity for trichloroethene hydrodechlorination," Industrial and Engineering Chemistry Research, vol. 48, no. 14, pp. 65506557, 2009.

[17] O. V. Kharissova, H. V. R. Dias, B. I. Kharisov, B. O. Pérez, and V. M. J. Pérez, "The greener synthesis of nanoparticles," Trends in Biotechnology, vol. 31, no. 4, pp. 240-248, 2013.

[18] N. Roy, A. Gaur, A. Jain, S. Bhattacharya, and V. Rani, "Green synthesis of silver nanoparticles: an approach to overcome toxicity," Environmental Toxicology and Pharmacology, vol. 36, no. 3, pp. 807-812, 2013.

[19] K. N. Thakkar, S. S. Mhatre, and R. Y. Parikh, "Biological synthesis of metallic nanoparticles," Nanomedicine: Nanotechnology, Biology, and Medicine, vol. 6, no. 2, pp. 257-262, 2010.

[20] S. Iravani, "Green synthesis of metal nanoparticles using plants," Green Chemistry, vol. 13, no. 10, pp. 2638-2650, 2011.

[21] Y. Park, Y. N. Hong, A. Weyers, Y. S. Kim, and R. J. Linhardt, "Polysaccharides and phytochemicals: a natural reservoir for the green synthesis of gold and silver nanoparticles," IET Nanobiotechnology, vol. 5, no. 3, pp. 69-78, 2011.

[22] C. Engelbrekt, K. H. Sørensen, J. Zhang, A. C. Welinder, P. S. Jensen, and J. Ulstrup, "Green synthesis of gold nanoparticles with starch-glucose and application in bioelectrochemistry," Journal of Materials Chemistry, vol. 19, no. 42, pp. 7839-7847, 2009.

[23] G. di Carlo, A. Curulli, R. G. Toro et al., "Green synthesis of gold-chitosan nanocomposites for caffeic acid sensing," Langmuir, vol. 28, no. 12, pp. 5471-5479, 2012.
[24] Z. Li, A. Friedrich, and A. Taubert, "Gold microcrystal synthesis via reduction of $\mathrm{HAuCl}_{4}$ by cellulose in the ionic liquid 1butyl-3-methyl imidazolium chloride," Journal of Materials Chemistry, vol. 18, no. 9, pp. 1008-1014, 2008.

[25] S. Pandey, G. K. Goswami, and K. K. Nanda, "Green synthesis of polysaccharide/gold nanoparticle nanocomposite: an efficient ammonia sensor," Carbohydrate Polymers, vol. 94, no. 1, pp. 229234, 2013.

[26] Y. Wang, L. Zhan, and C. Z. Huang, "One-pot preparation of dextran-capped gold nanoparticles at room temperature and colorimetric detection of dihydralazine sulfate in uric samples," Analytical Methods, vol. 2, no. 12, pp. 1982-1988, 2010.

[27] A. R. Choudhury, A. Malhotra, P. Bhattacharjee, and G. S. Prasad, "Facile and rapid thermo-regulated biomineralization of gold by pullulan and study of its thermodynamic parameters," Carbohydrate Polymers, vol. 106, no. 1, pp. 154-159, 2014.

[28] M. Amin, F. Iram, M. S. Iqbal, M. Z. Saeed, M. Raza, and S. Alam, "Arabinoxylan-mediated synthesis of gold and silver nanoparticles having exceptional high stability," Carbohydrate Polymers, vol. 92, no. 2, pp. 1896-1900, 2013.

[29] S. Ashraf, F. Sher, Z. M. Khalid, M. Mehmood, and I. Hussain, "Synthesis of cellulose-metal nanoparticle composites: development and comparison of different protocols," Cellulose, vol. 21, no. 1, pp. 395-405, 2014.

[30] T. Zhang, W. Wang, D. Zhang et al., "Biotemplated synthesis of cold nanoparticle-bacteria cellulose nanofiber nanocomposites and their application in biosensing," Advanced Functional Materials, vol. 20, no. 7, pp. 1152-1160, 2010.

[31] J. H. Johnston and T. Nilsson, "Nanogold and nanosilver composites with lignin-containing cellulose fibres," Journal of Materials Science, vol. 47, no. 3, pp. 1103-1112, 2012.

[32] A. Azetsu, H. Koga, L.-Y. Yuan, and T. Kitaoka, "Direct synthesis of gold nanocatalysts on TEMPO-oxidized pulp paper containing aldehyde groups," BioResources, vol. 8, no. 3, pp. 3706-3717, 2013.

[33] Technical Association of Pulp and Paper Industry, "Test Methods, T $200 \mathrm{sp}$-01, Laboratory beating of pulp (Valley beater method)," 2007.

[34] Technical Association of Pulp and Paper Industry, Test Methods, T 227 om-04, Freeness of Pulp (Canadian Standard Method), Technical Association of Pulp and Paper Industry, 2007.

[35] L. M. Liz-Marzán, "Nanometals: formation and color," Materials Today, vol. 7, no. 2, pp. 26-31, 2004.

[36] D. Philip, "Green synthesis of gold and silver nanoparticles using Hibiscus rosa sinensis," Physica E: Low-Dimensional Systems and Nanostructures, vol. 42, no. 5, pp. 1417-1424, 2010.

[37] A. Regiel, S. Irusta, A. Kyziol, M. Arruebo, and J. Santamaria, "Preparation and characterization of chitosan-silver nanocomposite films and their antibacterial activity against Staphylococcus aureus," Nanotechnology, vol. 24, no. 1, Article ID 015101, 2013.

[38] S. J. Yi, L. J. Xia, S. C. Lenaghan et al., "Bio-synthesis of gold nanoparticles using English ivy (Hedera Helix)," Journal of Nanoscience and Nanotechnology, vol. 13, no. 3, pp. 1649-1659, 2013.

[39] J. Pulit and M. Banach, "Preparation of nanosilver and nanogold based on Dog Rose aqueous extract," Bioinorganic Chemistry and Applications, vol. 2014, Article ID 658935, 14 pages, 2014.

[40] S. Y. Oh, I. Y. Dong, Y. Shin et al., "Crystalline structure analysis of cellulose treated with sodium hydroxide and carbon dioxide by means of X-ray diffraction and FTIR spectroscopy," Carbohydrate Research, vol. 340, no. 15, pp. 2376-2391, 2005. 
[41] I. K. Sen, K. Maity, and S. S. Islam, "Green synthesis of gold nanoparticles using a glucan of an edible mushroom and study of catalytic activity," Carbohydrate Polymers, vol. 91, no. 2, pp. 518-528, 2013.

[42] B. Cullity, Elements of X-Ray Diffraction, Addison-Wesley, London, UK, 1978.

[43] M. J. Richardson and J. H. Johnston, "Sorption and binding of nanocrystalline gold by Merino wool fibres-an XPS study," Journal of Colloid and Interface Science, vol. 310, no. 2, pp. 425430, 2007.

[44] L. K. Ono and B. R. Cuenya, "Formation and thermal stability of $\mathrm{Au}_{2} \mathrm{O}_{3}$ on gold nanoparticles: size and support effects," Journal of Physical Chemistry C, vol. 112, no. 12, pp. 4676-4686, 2008.

[45] H. Tsai, E. Hu, K. Perng, M. Chen, J.-C. Wu, and Y.-S. Chang, "Instability of gold oxide $\mathrm{Au}_{2} \mathrm{O}_{3}$," Surface Science, vol. 537, no. 1-3, pp. L447-L450, 2003.

[46] C.-M. Popescu, M.-C. Popescu, and C. Vasile, "Characterization of fungal degraded lime wood by FT-IR and 2D IR correlation spectroscopy," Microchemical Journal, vol. 95, no. 2, pp. 377-387, 2010.

[47] H. Wang, L. Huang, and Y. Lu, "Preparation and characterization of micro- and nano-fibrils from jute," Fibers and Polymers, vol. 10, no. 4, pp. 442-445, 2009.

[48] R. Li, M. He, T. Li, and L. Zhang, "Preparation and properties of cellulose/silver nanocomposite fibers," Carbohydrate Polymers, vol. 115, pp. 269-275, 2015.

[49] C. H. Ramamurthy, M. Padma, I. D. mariya samadanam et al., "The extra cellular synthesis of gold and silver nanoparticles and their free radical scavenging and antibacterial properties," Colloids and Surfaces B: Biointerfaces, vol. 102, no. 1, pp. 808-815, 2013.

[50] S. Medhe, P. Bansal, and M. M. Srivastava, "Enhanced antioxidant activity of gold nanoparticle embedded 3,6dihydroxyflavone: a combinational study," Applied Nanoscience, vol. 4, no. 2, pp. 153-161, 2014. 

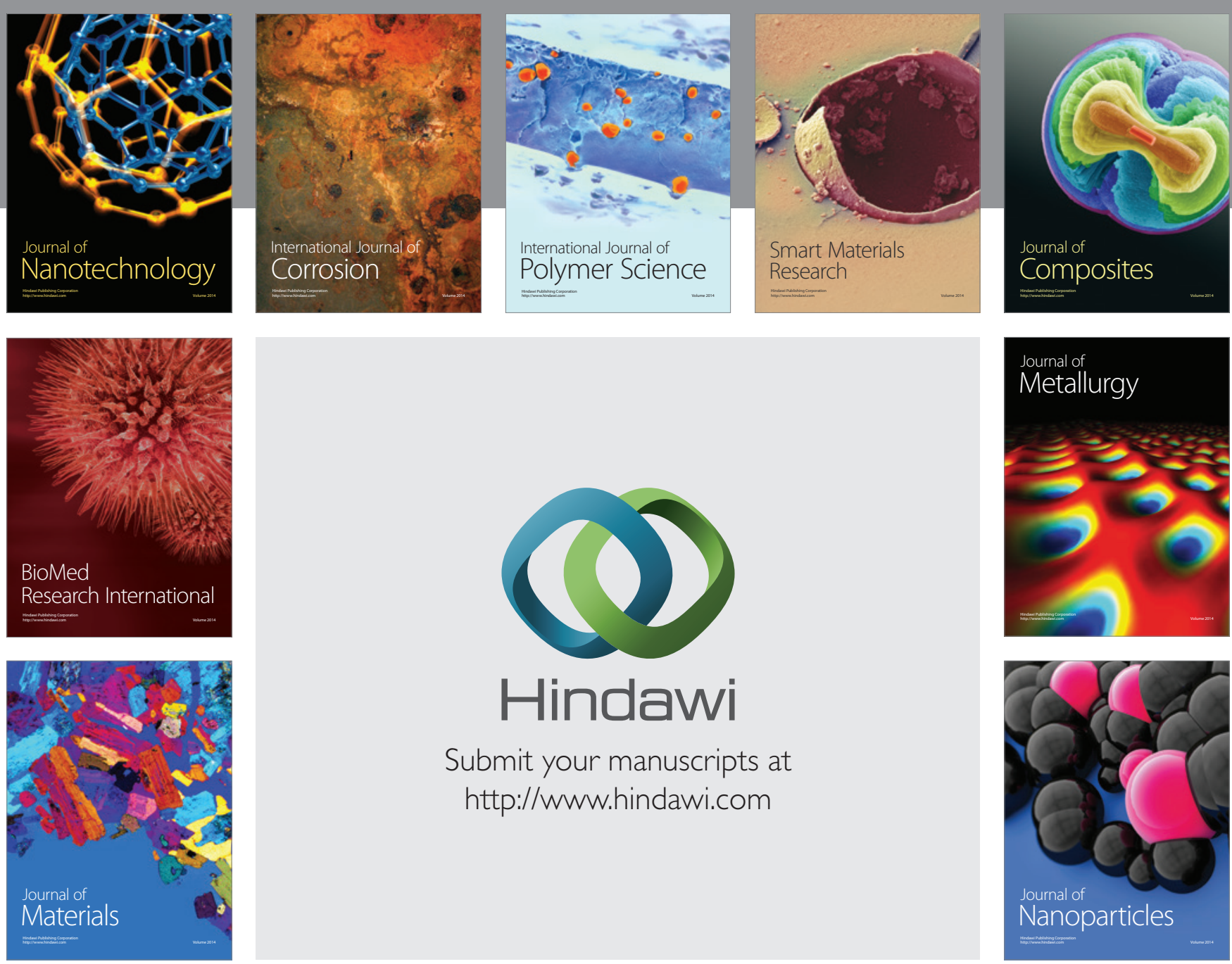

Submit your manuscripts at http://www.hindawi.com
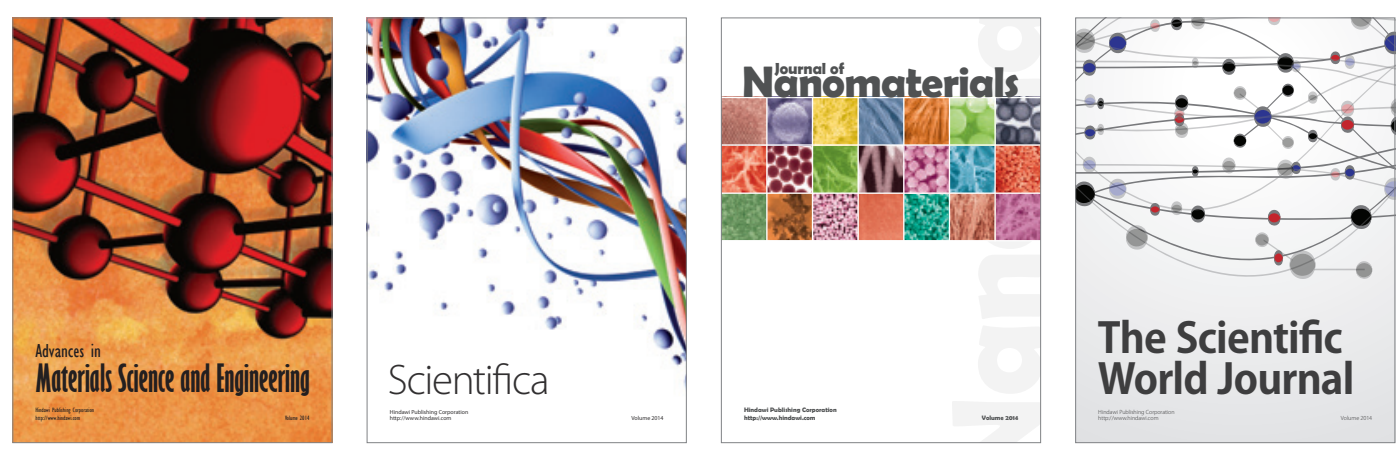

\section{The Scientific World Journal}
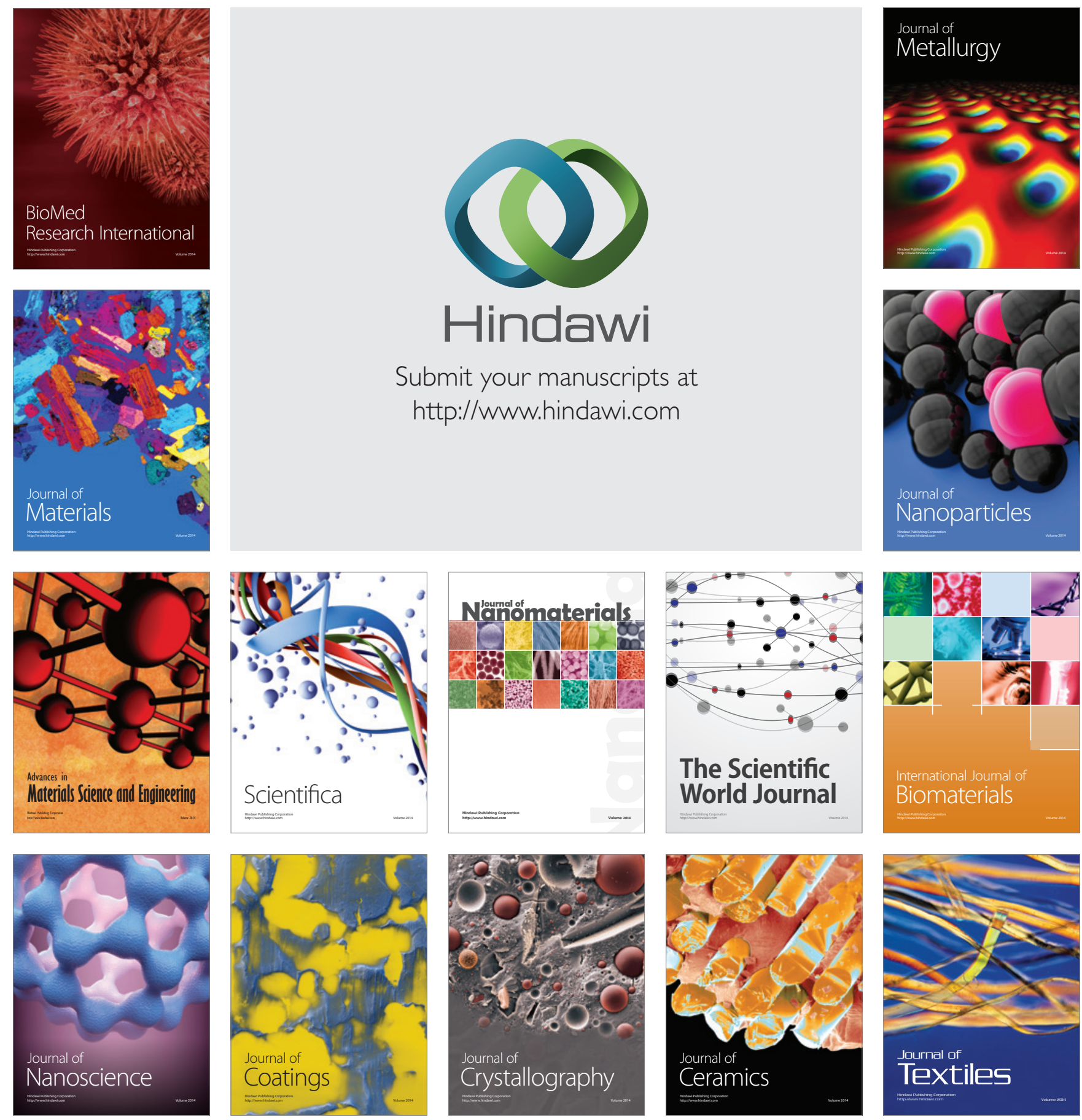